\title{
Size-arrival effects: The potential roles of conflicts between monocular and binocular time-to-contact information, and of computer aliasing
}

\author{
PATRICIA R. DELUCIA \\ Texas Tech University, Lubbock, Texas
}

\begin{abstract}
With computer simulations of self-motion, participants approached a floating object and tried to "jump" over it without collision. Participants "jumped" significantly later over small objects than they did over larger objects. This occurred when the displays were viewed monocularly or binocularly, a finding that suggests that such size-arrival effects (DeLucia \& Warren, 1994) were not due to a conflict between monocular and binocular cues to time-to-contact (TTC) information (Tresilian, 1994, 1995). Moreover, the results further suggest that size-arrival effects are not due to irregularities in TTC information that can occur from computer aliasing and that the latter does not always affect TTC estimation; visual information used in such judgments does not seem to be extracted on a frame-by-frame basis.
\end{abstract}

It was demonstrated in prior studies (DeLucia, 1989, $1991 \mathrm{a}, 1991 \mathrm{~b}$ ) that when computer-generated floating objects approached an observation point, observers reported that a large, far object appeared to hit the observation point before a small, near object appeared to hit the observation point, even though the small, near object was specified to arrive sooner by time-to-contact (TTC) information (tau: see, e.g., Lee, 1974, 1980). Such judgments were consistent with pictorial relative size rather than with TTC information and occurred even when motion-based information potentially exceeded threshold. Other results (DeLucia, 1991 a, 1991 b) indicated that such size-arrival effects were replicated with more textured objects (dotted square outlines similar to those used by Todd, 1981), relatively long display durations, and high-resolution photographic animations of approaching objects. Similar effects of pictorial relative size also occurred when observers judged whether two computer-simulated objects would collide with each other (DeLucia, 1995) and even occurred with an active collision-avoidance task (DeLucia $\&$ Warren, 1994). These results indicate that size-arrival effects can occur under a wide range of display and task conditions and suggest that judgments of TTC can be based

This material is based in part on work supported by the Texas Advanced Research Program under Grant No. 003644-017. It was also partly supported by the Research Enhancement Fund of Texas Tech University. I thank Jeff Maresh and Robert Todd of ESI for technical assistance and Heiko Hecht and two anonymous reviewers for comments on earlier versions. I am especially grateful to David A. Farcy for assistance with data collection and to Rik Warren for helpful discussions at earlier stages of Experiment 1. Correspondence concerning this article should be addressed to P. R. DeLucia, Department of Psychology, Texas Tech University, Lubbock, TX 79409-2051 (e-mail: djxpd@ttacs.ttu.edu).

-Accepted by previous editor, Myron L. Braunstein on information other than, or in conjunction with, optical TTC (see also, e.g., DeLucia \& Novak, 1997; Kaiser \& Mowafy, 1993).

However, Tresilian (1995; see also Tresilian, 1994) pointed out that because observers viewed the "monocular" displays binocularly, there was a conflict between monocular and binocular TTC information, which may account for size-arrival effects on active collision avoidance. Specifically, he proposed that binocular TTC information indicated that the targets were stationary (or that the rate of approach was too slow for the information to be above threshold) and that only the changing image size was a source of TTC information. On the basis of Heuer's (1993) research, Tresilian further proposed that the visual tau values of relatively large objects (i.e., those with larger image sizes) contributed more to TTC estimates than did the visual tau values of smaller objects. Similarly, those information sources which indicated that the object was not approaching contributed more to estimates when the image sizes were relatively small, which led to the sizearrival effect. Therefore, he predicted that TTC judgments would not be affected by image size if the displays were viewed monocularly.

On the other hand, Tresilian (1995) also noted that such a conflict could not account for size-arrival effects in the relative TTC judgments observed by DeLucia (1991a) because observers viewed the displays monocularly. Thus, he hypothesized that such relative TTC judgments involved the cognitive visual system, whereas the active collisionavoidance task (DeLucia \& Warren, 1994) involved the motor visual system. That is, the explanation of sizearrival effects depends on the task (Tresilian, 1995).

Moreover, Gray and Regan (1996; see also Gray \& Regan, 1998) reported that, when only binocular information was available, errors in absolute TTC estimates of 
simulated approaching objects were small. With only monocular information, errors were small for objects with relatively small starting sizes but were larger for the largest starting size. That is, monocular information can result in an effect of the object's starting size. When binocular and monocular information were available, judgments were more accurate and there was no effect of starting size. Further, monocular information may be more important than binocular information as object size increases (Regan, 1997). The implications for the present study are that the potential effect of a conflict between monocular and binocular TTC information on TTC estimates may depend on the object's size and that such a conflict may contribute to size-arrival effects. Therefore, it is important to determine whether size-arrival effects on collision avoidance can be eliminated with monocular viewing. (For other TTC studies that compared performance between monocular and binocular conditions, see Cavallo \& Laurent, 1988; Kebeck \& Landwehr, 1992; Michaels, Zeinstra, \& Oudejans, 1997; and Savelsberg, Whiting, \& Bootsma, 1991.)

It also is important to consider the possibility that computer aliasing ${ }^{\prime}$ affects TTC estimates and contributes to size-arrival effects because low-resolution displays may not simulate 3-D environments adequately (Tresilian, personal communication, September 7, 1995). Moreover, the effects of aliasing may vary with an object's optical size. DeLucia (1991a) observed that aliasing can affect the precision of optical TTC information but concluded from empirical data that the potential effects of computer aliasing on TTC information do not account for sizearrival effects in relative TTC tasks. In this study, the effects of computer aliasing are further examined by means of a prediction motion (PM) task (for related work, see Hecht, Kaiser, Savelsbergh, \& van der Kamp, 1996). The results have methodological and theoretical implications for studies of TTC estimation because it is not clear how often the perceptual system samples TTC information. If observers extract TTC on a frame-by-frame basis (i.e., with a fast sampling rate), imprecisions in TTC information produced by factors such as aliasing may affect TTC estimates. Alternatively, observers may use an averaging process to extract TTC, and it is therefore important to determine the size of the "averaging window" (see also DeLucia, 1991a; Tresilian, 1993).

In Experiment 1, active collision avoidance was measured during monocular viewing and binocular viewing. In Experiment 2, TTC estimation was measured with a PM task under different levels of (simulated) aliasing. It is shown that such factors do not seem to account for size-arrival effects.

\section{EXPERIMENT 1}

\section{Method}

Participants. The participants were 8 male and 8 female students at Texas Tech University who received credit in a psychology course for their participation in the experiment, had normal or correctedto-normal visual acuity, and were not informed of the experimental hypotheses.
Apparatus and Displays. Computer simulations were created with a Pentium $90-\mathrm{MHz}$ computer and were presented in $640 \times 350$ pixel resolution at a speed of 35 frames $/ \mathrm{sec}$ on a 14 in. $(35.56 \mathrm{~cm})$ monitor. The scenes represented self-motion toward a square object. For simplicity. lengths are described in feet and meters, but rescaling to other units is possible. The parameters of the scenes approximated those in Experiment 1 of De Lucia and Warren (1994). However, the displays in this study were simpler in that the objects consisted of square black outlines inscribed with a cross and were shown against a white background. ${ }^{2}$ (See Figure 1.) Although the lines that defined the square and cross increased in length during approach, they did not increase in thickness. ${ }^{3}$ The virtual eye (self) always began at an altitude of $128 \mathrm{ft}(38.78 \mathrm{~m})$ and at a distance of $1,280 \mathrm{ft}(387.84 \mathrm{~m})$ from a single square; self-motion toward the square was always constant at $150 \mathrm{ft} / \mathrm{sec}(45.45 \mathrm{~m} / \mathrm{sec})$. The size of the virtual square was $16 \mathrm{ft}(4.85 \mathrm{~m}), 72 \mathrm{ft}(21.82 \mathrm{~m})$, or $128 \mathrm{ft}$ $(38.78 \mathrm{~m})$. Thus, at the beginning of the trial, the squares subtended approximately $.72^{\circ}, 3.22^{\circ}$, and $5.73^{\circ}$ of visual angle, respectively.

Procedure and Design. The methods approximated those reported in an earlier study (DeLucia \& Warren, 1994) and are summarized here. The participants' chins were supported by a chinrest, and they viewed the displays in a dimly lit room, at the projectively correct viewing position (from a distance of $36 \mathrm{~cm}$ ). They were instructed to jump over the square and come back down behind the square (i.e., to follow the contour of the square); they were instructed to get as close as possible to the square without hitting it. The participants pulled a control stick backward (toward them) in order to ascend and pushed it forward (away from them) in order to descend; the maximum vertical velocity was $100 \mathrm{ft} / \mathrm{sec}(30.3 \mathrm{~m} / \mathrm{sec})$. Altitude control was possible at the start of the trial and lasted for $9.86 \mathrm{sec}$; altitude was sampled at approximately $35 \mathrm{~Hz}$ and was constant during stick motion. ${ }^{4}$ The time between the start of the trial and potential contact with the square was $8.53 \mathrm{sec}$. A "dead zone" of $5 \%$ was set on the stick to minimize drift due to noise. After the observers completed their response. they pressed a button to end the trial.

it was reasonable to expect that the height of the square would affect the initiation of the jump. Thus, it was essential to compare jumps for different-sized squares only when the tops of the squares were at the same height. For each scene in which a square was centered on the principal line of sight, separate scenes were created in which the other squares' heights were adjusted so that all the square tops were aligned. There were three levels of this square-top factor, one for each square size. Thus, there were nine unique scenes, each replicated 10 times in a random order; half of the observers viewed the reverse order. Feedback was not provided, and intertrial duration was self-paced. Six practice trials were provided (more upon request) to familiarize the participants with the task and the dynamics of the control stick; the practice trials were not included in the analyses.

Finally, half the observers had the left eye covered with an eye patch, and half viewed the displays with both eyes. The primary objective of Experiment 1 was to determine whether size-arrival effects can occur with monocular viewing. A binocular viewing condition was included to permit a direct comparison with monocular view-
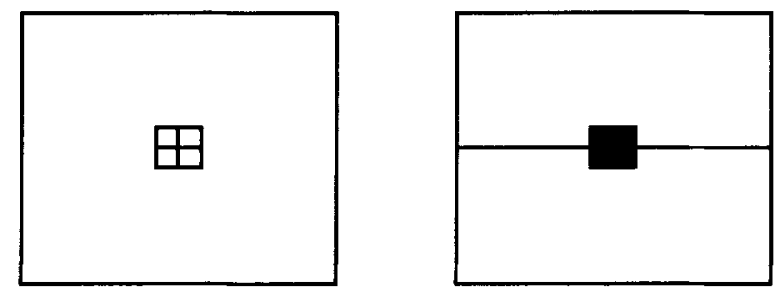

Figure 1. Schematic representation of scenes. Left panel: Experiment 1. Right panel: Experiment 2. Motion was along the depth axis. 
ing and with the pattern of results in the earlier study (DeLucia \& Warren, 1994).

Square outlines were used instead of filled-in squares (described by DeLucia \& Warren, 1994) because of technical considerations. When a display simulates an observer's approach toward a filled-in square, the square's optical size increases and the computer requires more time to draw it. The available computer was not fast enough to draw such scenes and to sample altitude in real time simultaneously, a condition that is necessary to permit interactive control. This limitation (also an issue when texture is added to the squares) was avoided with the simpler square outlines. Moreover, previous results indicated that size-arrival effects on a PM task can occur with square outlines inscribed with a cross (DeLucia \& Warren, 1994).

\section{Results}

The main objective of Experiment 1 was to determine whether the size-arrival effects that previously occurred with a collision-avoidance task (DeLucia \& Warren,
1994) would occur with monocular viewing (i.e., when there was no conflict between binocular and monocular cues to TTC information). To determine whether observers jumped sooner to avoid collision with larger squares than smaller squares, measures of launch time were used. Launch time was defined as the amount of time that elapsed after the beginning of the trial when the jump was initiated, ${ }^{5}$ and it is the focus of my discussion. To provide interested readers with information about other dependent variables that were reported in the previous study on collision avoidance (DeLucia \& Warren, 1994), several of these measures are included in Figures 2-3 and in Table 1: the maximum altitude that occurred after $6 \mathrm{sec}$ from the beginning of the trial, or the peak altitude (a 6-sec criterion was used to exclude early false jumps); the time at which the peak altitude occurred, or the time at peak altitude 6 ; altitude at the time of the launch; the
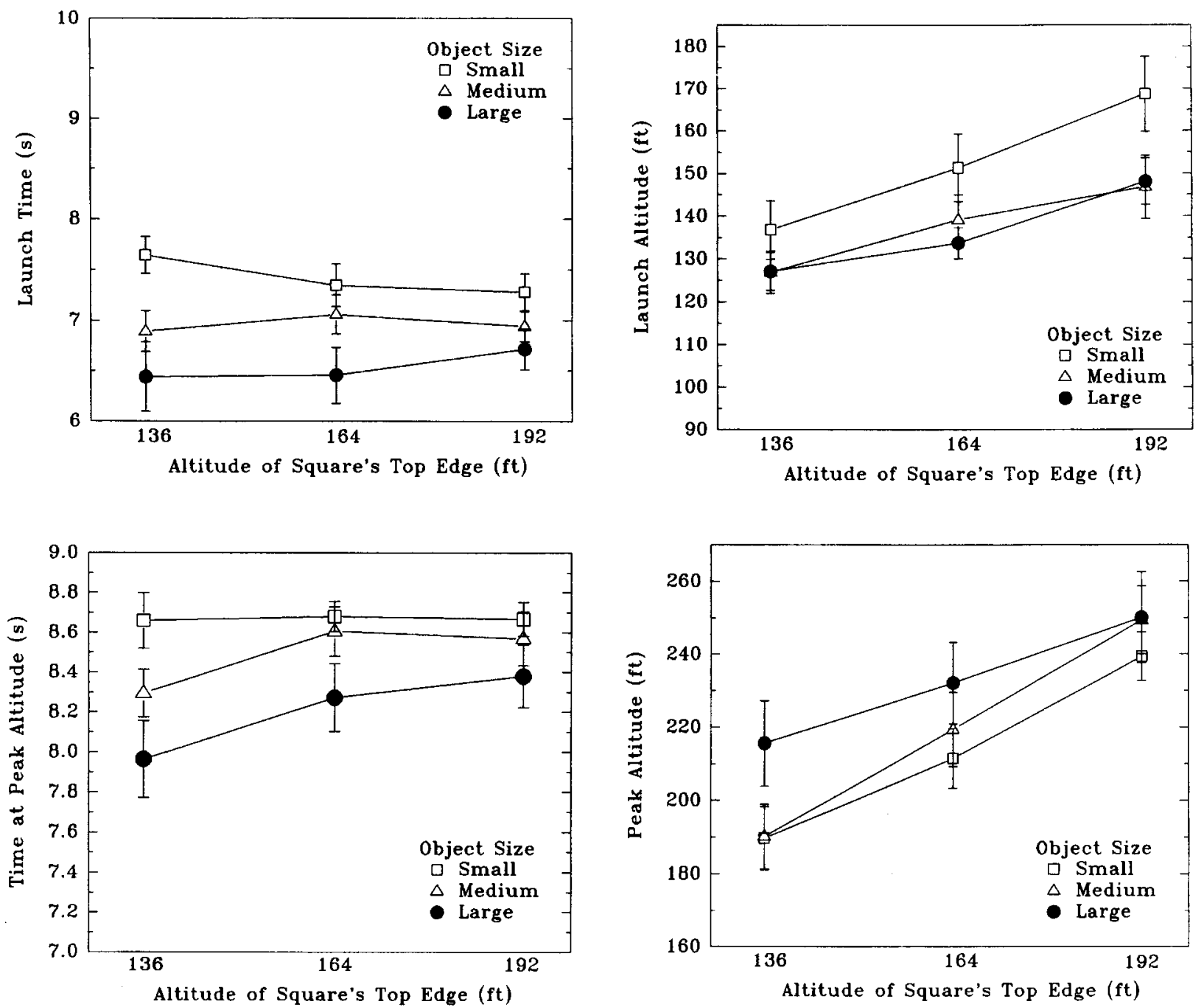

Figure 2. Experiment 1, Monocular viewing. Means of launch time, launch altitude, time at peak altitude, and peak altitude, as a function of square top and square size. Error bars indicate $\pm 1 S E M$. 

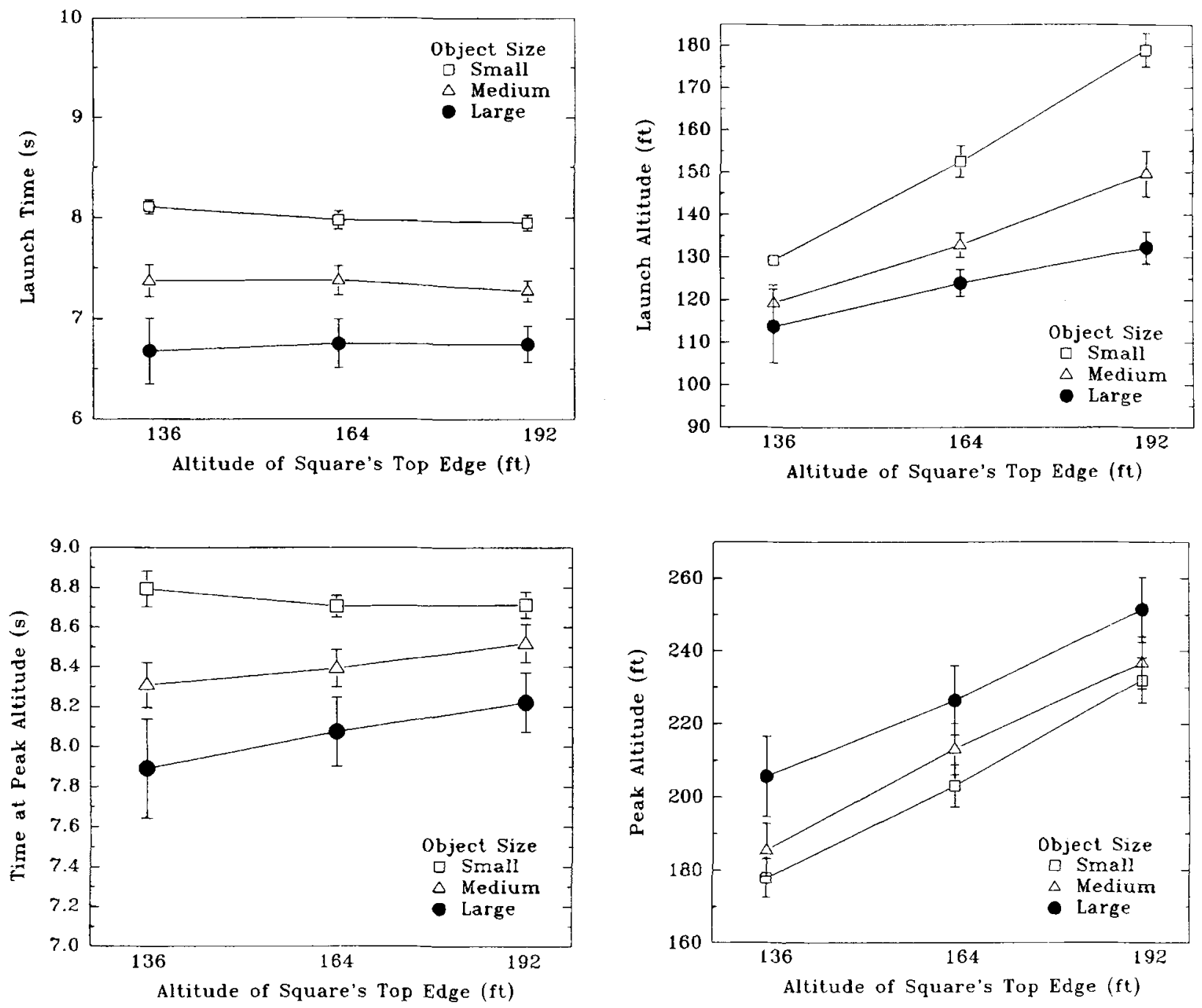

Figure 3. Experiment 1, Binocular Viewing. Means of launch time, launch altitude, time at peak altitude, and peak altitude, as a function of square top and square size. Error bars indicate $\pm 1 S E M$.

difference in altitude between the self and the top of the square when the self was directly over the square, or the clearance; and the number of trials in which the self and the square were at the same place at the same time (expressed as a percentage of the trials), or the frequency of crashes. There were no significant effects that involved clearance or crashes, which will not be included.

The results of monocular and binocular viewing are summarized in Figures 2 and 3, respectively, and were analyzed with a $2 \times 3 \times 3$ (ocularity $\times$ square size $\times$ square top) mixed analysis of variance (ANOVA). ${ }^{7}$ Two observers were replaced because they consistently jumped under the square and may have held the stick upside down. For brevity, the $F, p$, and $\omega^{2}$ values are shown in Table 1 . Tukey's HSD test and an alpha level of .05 were used for all post hoc comparisons.
Launch time. Only square size affected launch time, which increased as size decreased. All pairwise comparisons were significant the means were $7.72 \mathrm{sec}, 7.15 \mathrm{sec}$, and $6.63 \mathrm{sec}$, respectively). (The corresponding means reported by DeLucia \& Warren, 1994, were $7.80 \mathrm{sec}$, $7.39 \mathrm{sec}$, and $6.89 \mathrm{sec}$ in Experiment 1, and 7.94, 7.49, and 7.15 in Experiment 2.)

Altitude at launch. There were main effects of square top and square size. Launch altitude was greater when square size was $16 \mathrm{ft}$ than when it was either $72 \mathrm{ft}$ or $128 \mathrm{ft}$, and launch altitude increased as square top increased; all pairwise comparisons were significant. There was also a significant interaction between square size and square top.

Time at peak altitude. There were effects of square top and square size. Time at peak aititude increased as 
Table 1

Results of Experiment 1

\begin{tabular}{|c|c|c|c|c|c|c|c|c|c|c|c|c|}
\hline \multirow[b]{2}{*}{ Dependent Variable } & \multicolumn{4}{|c|}{ Square Size } & \multicolumn{4}{|c|}{ Square Top } & \multicolumn{4}{|c|}{ Top $\times$ Size } \\
\hline & $F$ & $d f$ & $p<$ & $\omega^{2}(\%)$ & $F$ & $d f$ & $p<$ & $\omega^{2}(\%)$ & $F$ & $d f$ & $p<$ & $\omega^{2}(\%)$ \\
\hline \multicolumn{13}{|c|}{ Monocular and Binocular Viewing } \\
\hline Launch time & 34.64 & 2,28 & .0001 & 35.41 & & & n.s. & & & & n.s. & \\
\hline Launch altitude & 27.75 & 2,28 & .0001 & 18.99 & 48.97 & 2,28 & .0001 & 27.31 & 7.66 & 4,56 & .0003 & 2.41 \\
\hline Time at peak altitude & 21.15 & 2,28 & .0002 & 25.44 & 14.34 & 2,28 & .0004 & 3.05 & 3.40 & 4,56 & .0330 & 1.78 \\
\hline Peak altitude & 17.44 & 2,28 & .0004 & 6.86 & 198.35 & 2,28 & .0001 & 36.93 & 2.92 & 4,56 & .0426 & 0.45 \\
\hline \multicolumn{13}{|c|}{ Monocular Viewing } \\
\hline Launch time & 8.08 & 2,14 & .0206 & 22.42 & & & n.s. & & & & & \\
\hline Launch altitude & 6.07 & 2,14 & .0029 & 9.48 & 21.24 & 2,14 & .001 & 20.30 & & & & \\
\hline Time at peak altitude & 7.85 & 2,14 & .0167 & 16.16 & 14.15 & 2,14 & .0024 & 5.32 & & & & \\
\hline Peak altitude & 7.38 & 2,14 & .0153 & 4.15 & 65.57 & 2,14 & .0001 & 31.02 & & & & \\
\hline \multicolumn{13}{|c|}{ Binocular Viewing } \\
\hline Launch time & 42.28 & 2,14 & .0002 & 52.76 & & & n.s. & & & & n.s. & \\
\hline Launch altitude & 30.26 & 2,14 & .0007 & 29.56 & 27.75 & 2,14 & .0005 & 33.65 & 11.68 & 4,28 & .001 & 4.98 \\
\hline Time at peak altitude & 13.54 & 2,14 & .0059 & 32.27 & & & n.s. & & & & n.s. & \\
\hline Peak altitude & 10.11 & 2,14 & .0148 & 8.75 & 184.47 & 2.14 & .0001 & 42.54 & & & n.s. & \\
\hline
\end{tabular}

Note-For monocular and binocular viewing, ocularity was not significant. For monocular viewing, interactions were not significant.

square size decreased; all pairwise comparisons were significant. Peak altitude was greater when square top was $192 \mathrm{ft}$ or $164 \mathrm{ft}$ than when it was $136 \mathrm{ft}$. There was also a significant interaction between square size and square top.

Peak altitude. There were main effects of square top and square size. Peak altitude was greater when the square size was $128 \mathrm{ft}$ than when it was $16 \mathrm{ft}$ or $72 \mathrm{ft}$, and increased as square top increased; all pairwise comparisons were significant. There was also a significant interaction between square size and square top.

The results indicated that there were no significant effects involving ocularity. However, the latter was a between-groups factor, and the absence of significant effects may be attributable partly to variability among participants. To determine whether square size significantly affected launch time when viewing was monocular or binocular, I also conducted separate $3 \times 3$ (square size $\times$ square top) repeated measures ANOVAs on the two viewing conditions. With monocular viewing, only square size affected launch time, which was greater when the square was $16 \mathrm{ft}$ than when it was $128 \mathrm{ft}$. The means were $7.42 \mathrm{sec}, 6.96 \mathrm{sec}$, and $6.54 \mathrm{sec}$ for $16-\mathrm{ft}, 72-\mathrm{ft}$, and $128-\mathrm{ft}$ squares, respectively. With binocular viewing, only square size affected launch time, which increased as square size decreased; all comparisons were significant. The respective means were $8.01 \mathrm{sec}, 7.34 \mathrm{sec}$, and $6.73 \mathrm{sec}$. Thus, in both viewing conditions, observers approached the smaller squares more closely before jumping to avoid collision and jumped sooner when they approached larger squares.

In summary, size-arrival effects occurred when observers viewed the displays with either one eye or both eyes. ${ }^{8}$ Therefore, the results suggest that a conflict between monocular and binocular TTC information does not account for size-arrival effects in the collision-avoidance task, at least in the conditions studied here. However, the data shown in Figures 2 and 3 suggest greater response variability with monocular viewing, a finding that deserves further study.

\section{POTENTIAL EFFECTS OF COMPUTER ALIASING}

The issue of how well low-resolution displays are able to simulate virtual environments is an important consideration (Tresilian, personal communication, September 7, 1995) that is not typically addressed in TTC studies (but see DeLucia, 1991a). The digitization process of computers produces aliasing, which can lead to artifacts in computer simulations of 3-D scenes. Specifically, intensities from a continuous image space (the 3-D image space or the 2-D projection of that image space) are mapped to pixels in discrete image space; in essence, a continuous space is sampled with discrete sample points (Watt, 1989). Simulations of moving environments also contain undersampling in the temporal domain (Watt, 1989).

The effect of aliasing is not limited to the familiar jagged edges in, for example, diagonal lines. It also affects the accuracy of a simulated object's projected size, because the computer must round to the nearest integer in order to determine which pixel to illuminate; a fraction of a pixel cannot be illuminated. In a simulation of an approaching object, increases in the object's computer-generated image from one frame, or one point in time, to the next may be too large or too small (compared with an ideal projection medium) because of the digitization process; increases that are less than the size of a pixel cannot be represented at all. Such artifacts of aliasing can potentially affect observers' judgments about the computer-generated scenes, and this effect is exacerbated in displays with relatively low resolution. Thus, the potential contribution of aliasing to TTC judgments and size-arrival effects deserves consideration. To consider such effects, I use a "worst- 
case scenario," in which the image of a computer-simulated approaching object does not change in every frame.

It is relevant to note that the effects of aliasing on TTC judgments would occur only if the inaccuracies in the simulation are within the spatiotemporal resolution of the perceptual system. For example, if an approaching object's optical size does not increase from one frame to the next, the optical TTC information is not accurate for that time period. However, it is not clear how often the perceptual system samples optical TTC information; in other words, it is not clear whether the system extracts TTC on a frame-by-frame basis or extracts TTC by averaging optical expansion information over several frames (see also DeLucia, 1991a; Tresilian, 1993). If TTC is extracted by averaging, simulation errors that occur between two successive frames may not be a critical factor in TTC judgments.

It seems unlikely that arrival time is perceptually extracted frame by frame: Because of the spatiotemporal limits of the visual system, the minimum time over which temporal information is integrated before it can be used may range from $32 \mathrm{msec}$ (Morgan, 1980) to $350 \mathrm{msec}$ (Smith \& Gulick, 1957), depending on the display and task (DeLucia, 199 la). That observers do not extract TTC on a frame-by-frame basis is suggested by the results of an earlier study (DeLucia, 1991a). Computer simulations of two objects that approached the observation point were created with $320 \times 200$ pixel resolution and a presentation rate of 30 frames $/ \mathrm{sec}$. The observers reported that a large, far approaching object appeared to hit the observation point before a small, near object appeared to hit the observation point, even though the small, near object was specified to arrive sooner by optical TTC information; a size-arrival effect occurred. However, when the absolute TTC of both objects was reduced by decreasing their initial distances to the observation point (optical expansion of both objects was greater than in the original scene), observers reported correctly that the small, near object would arrive first. Their judgments were consistent with relative TTC information. Consequently, this scene was manipulated to simulate an exaggerated level of aliasing to determine whether the corresponding increase in irregularities in TTC information would affect TTC judgments. Four of the 10 frames of the scene were shown three times in succession, which resulted in substantial irregularities in TTC information (see DeLucia, 1989, for details). Nevertheless, observers judged relative TTC accurately, which suggests that irregularities in TTC information were not critical to observers when making their judgments, and that observers did not extract optical TTC on a frame-by-frame basis.

Furthermore, it is important to note that, owing to visual thresholds, observers do not necessarily use optical TTC information more effectively with high-resolution displays than with low-resolution displays. It was noted earlier that the effects of pictorial size on judgments of relative TTC that were obtained with low-resolution computer displays persisted when the resolution was in- creased substantially with film animations. Moreover, the amount of change in an approaching object's optical size does not depend solely on the spatial resolution of the display; it also depends on the object's virtual distance, size, and velocity. Even with film animation (or a realworld scenario), the increase in an approaching object's optical size from one point in time to the next could be below visual thresholds if the object is small, is distant from the eye, and moves slowly.

An analysis of the scenes described in Schiff and Detwiler (1979) indicated that this was the case in their study. To study TTC, they used film animations of an approaching object, projected at 24 frames/sec (see their Table 1, Experiment 3, small object, far final distance). In scenes that simulated an 8-sec TTC, for example, the object's optical size increased from approximately $.688^{\circ}$ to $.859^{\circ}$ over a duration of $2 \mathrm{sec}$, or 48 frames. An analysis of the object's optical size in each frame (using the information provided in their Table 1 and assuming a continuous projection medium) indicated that the largest expansion that occurred in any two successive frames was only .30-min arc. This is below the previously reported thresholds for size change from simulated movement in depth (about I-min arc reported in Hills, 1975; see also Baker \& Steedman, 1961). Even when TTC was reduced to $4 \mathrm{sec}$, the largest frame-by-frame expansion was $.54 \mathrm{~min}$. Nevertheless, the results of Schiff and Detwiler's study indicated that estimated TTC increased as actual TTC increased; the observers' judgments were consistent with the use of optical TTC information (albeit judgment accuracy decreased as TTC increased). This suggests that observers did not extract TTC on a frameby-frame basis, possibly because of limits in the temporal resolution of the visual system (for related discussions, see Hochberg, 1986).

The potential effects of aliasing on TTC estimation have several important implications. First, aliasing can produce inaccuracies in the projected size of an approaching object from one frame to the next and thereby can affect TTC information specified by any two frames. Indeed, an earlier analysis indicated that even small inaccuracies in an image can have substantial effects on TTC information (DeLucia, 1991a). As noted before, it seems unlikely that observers extract TTC on a frame-by-frame basis. It then becomes important to determine whether observers use an averaging process to extract TTC and, if so, to determine the size of the "averaging window" (see also DeLucia, 1991a: Tresilian, 1993).

Moreover, aliasing can contribute to size-arrival effects if objects with small projected images are affected more by aliasing than are objects with larger projected images. For example, at one extreme, objects that are relatively small (spatial extent is less than the area of a pixel) may appear and disappear during animated approach motion (Watt, 1989). Further, the change in a small object's image during simulated approach is smaller than the change in a larger object's image (assuming the same virtual velocities and distances). Thus, in a given frame, 
inaccuracies in optical expansion due to aliasing will likely make up a relatively greater proportion of the total optical expansion in objects with smaller projected sizes. If aliasing affects simulations of approach in this manner, it could contribute to size-arrival effects.

In prior studies, the potential contribution of irregularities in TTC information to size-arrival effects was evaluated with measures of relative TTC judgments (DeLucia, 1991a). In the present study, such effects of aliasing were measured with a PM task, which may involve cognitive motion extrapolation (DeLucia \& Liddell, 1998; Schiff \& Oldak, 1990; Tresilian, 1995).

\section{EXPERIMENT 2}

The first aim of Experiment 2 was to evaluate the potential effects of aliasing on absolute TTC judgments in a PM task. Aliasing was manipulated with the method described by DeLucia (1989, 1991a), who measured relative TTC judgments. The second aim of Experiment 2 was to determine whether differential effects of aliasing on objects of different sizes contribute to size-arrival effects; comparisons were made between TTC estimates of small and large objects with the same TTC but different degrees of aliasing.

\section{Method}

Displays. Computer simulations were created with an MS-DOS Pentium-90 MHz computer with $640 \times 350$ pixel resolution and a presentation rate of 23.3 frames/sec. Displays consisted of perspective black-on-white drawings of 3-D scenes in which a square object approached the observation point while centered vertically on a horizon line. The scenes were similar to a subset of those described by Schiff and Detwiler (1979; plain white terrain, plain sky). (See Figure 1 and Table 2.)

Displays were created by simplifying a subset of scenes described in an earlier paper (DeLucia \& Liddell, 1998, control study, fardistance scenes only; thus, when TTC increased, velocity decreased). At the beginning of each scene, a small object that was $100 \times 100 \mathrm{ft}$

Table 2

Experiment 2: Degrees of Visual Angle Subtended by the Object's Height (Large Objects Unless Noted)

\begin{tabular}{|c|c|c|c|}
\hline \multirow{2}{*}{$\begin{array}{c}\text { TTC of } \\
\text { Virtual Scene } \\
\text { (Seconds) }\end{array}$} & \multirow{2}{*}{$\begin{array}{l}\text { Percentage } \\
\text { Frames With } \\
\text { No Motion }\end{array}$} & \multicolumn{2}{|c|}{ Visual Angle } \\
\hline & & $\begin{array}{l}\text { First } \\
\text { Frame }\end{array}$ & $\begin{array}{c}\text { Last } \\
\text { Frame }\end{array}$ \\
\hline 1.06 & 0 & 4.92 & 14.19 \\
\hline 1.10 & 19 & 4.92 & 14.19 \\
\hline 1.10 & 45 & 4.92 & 13.64 \\
\hline 1.14 & 74 & 4.92 & 13.14 \\
\hline $1.06^{*}$ & 43 & .82 & 2.38 \\
\hline 4.52 & 0 & 9.86 & 14.21 \\
\hline 4.52 & 19 & 9.86 & 14.21 \\
\hline 4.56 & 45 & 9.86 & 14.10 \\
\hline 4.60 & 74 & 9.86 & 13.95 \\
\hline $4.52^{*}$ & 64 & 1.65 & 2.38 \\
\hline 6.62 & 0 & 11.70 & 15.21 \\
\hline 6.62 & 19 & 11.70 & 15.21 \\
\hline 6.67 & 45 & 11.70 & 15.11 \\
\hline 6.71 & 74 & 11.70 & 15.02 \\
\hline $8.47^{*}$ & 77 & 1.92 & 2.38 \\
\hline
\end{tabular}

*Small object.
( $30.48 \mathrm{~m}$ ) was stationary for $1 \mathrm{sec}$; it then moved at a constant speed for $2 \mathrm{sec}$ and disappeared when it was $1.1 \mathrm{sec}, 4.5 \mathrm{sec}$, or $8.5 \mathrm{sec}$ from virtual contact with the observation point. Additional scenes were created by increasing the object's virtual size by sixfold; this resulted in a perceptible change in its optical size in every frame ${ }^{9}$ These scenes are considered to have $0 \%$ aliasing. However, to create $0 \%$ aliasing in the scenes with an 8.5-sec TTC, the object's velocity also had to be increased by about $19 \%$. As a result, in the scenes with the larger objects, the TTC to the observation point was about $6.6 \mathrm{sec}$. However, a comparison of TTC estimation between the scenes with the small and large objects that had TTC values of $1.1 \mathrm{sec}$ and $4.5 \mathrm{sec}$ provided a measure of whether pictorial size affects TTC estimation and whether aliasing contributes to size- arrival effects.

The scenes were manipulated with animation techniques. The object's motion was paused in selected frames so that its optical size did not change in $19 \%, 45 \%$, or $74 \%$ of the frames ( 47 total). By doing so, I simulated different degrees of aliasing or "jerkiness" in the object's optical expansion and thus its frame-by-frame TTC. The frames in which the object's motion was paused were selected by first analyzing approach scenes in the control study of DeLucia and Liddell (1998). To create three levels of simulated aliasing or irregularities in the optical expansion pattern, I determined in which frames the object's optical size did not change because of computer aliasing. I then applied this pattern to the scenes used in the present study; three patterns were used in Experiment 2.

Frames 1-23 made up the static portion of the scenes. In scenes with $0 \%$ aliasing, the object's optical size increased in Frames 24-70 (the entire period of motion). With aliasing in 19\% of the frames, there was no change in the object's optical size in Frames $24,27,29,34,37,39,41,43$, and 50 . In the $45 \%$ condition, there was no change in optical size in Frames 26-28, 30-32, 34-36, 38-39, $42,45-46,49,52,55,58,61,65$, and 70 . In the $74 \%$ condition, there was no change in optical size in Frames 24-26, 28-34, 36-37, $39-41,43-47,49,51-54,57-60,63-65,67$, and 69-70. When the optical size did not change in the last frame, the TTC varied by a small amount (when computed with visual angles on the first and last frames; see Table 2). Finally, in the small-object scenes, the object's optical size did not change in $43 \%, 64 \%$, and $77 \%$ of the frames when TTC was $1.1 \mathrm{sec}, 4.5 \mathrm{sec}$, and $8.5 \mathrm{sec}$, respectively.

The five types of scenes (small-object scenes and four large-object scenes with different levels of aliasing) and different levels of TTC resulted in 15 unique scenes. Each was presented five times in one of two random orders; order was counterbalanced across observers.

Finally, a filled-in square was used in Experiment 2, rather than the square outlines used in Experiment 1 , because the observers did not have to jump over the squares; the scene ended before the object's image size became too large to create timing problems. Furthermore, size-arrival effects on collision avoidance were obtained with filled-in squares (DeLucia \& Warren, 1994) and with square outlines (in Experiment 1 of the present study) and with square outlines and a PM task (DeLucia \& Warren, 1994). These results indicate that the design of the squares does not have significant consequences for such effects (see also DeLucia, 199lb).

Procedure. Observers viewed the displays with both eyes from a distance of $2 \mathrm{ft}(.61 \mathrm{~m})$. They were instructed to press a mouse button when they thought that the object would reach them had it kept approaching at the same speed after it disappeared. Fifteen practice trials were provided but were not analyzed. Finally, it was not feasible to use the collision-avoidance task that was used in Experiment 1 . To evaluate the effects on performance of irregularities in TTC information due to simulated aliasing, it was necessary to control the image size of the objects in each frame. An interactive task might have interfered with this control.

\section{Results and Discussion}

Figure 4 shows that the degree of simulated aliasing did not produce systematic effects on TTC estimates. 


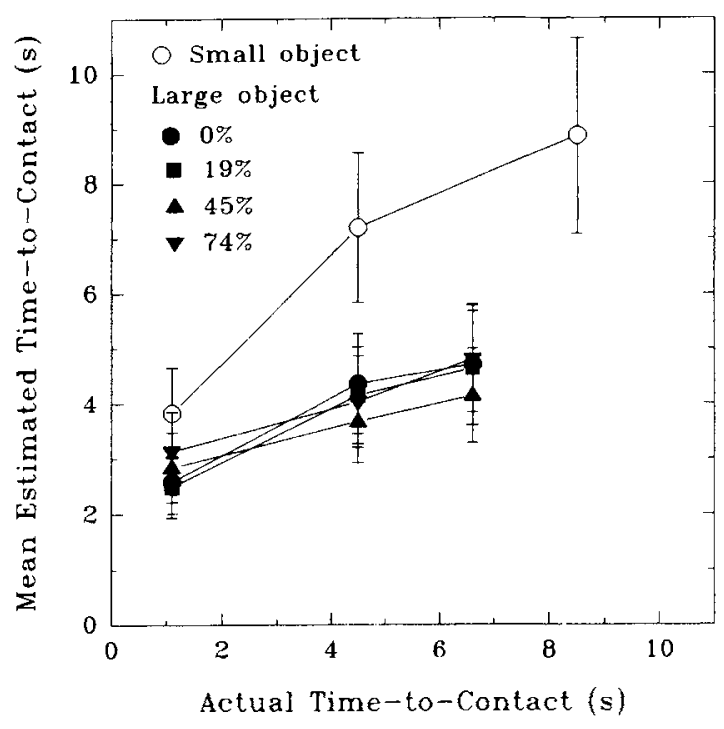

Figure 4. Experiment 2. Mean TTC estimation as a function of actual TTC for each level of simulated aliasing and object size. Error bars indicate \pm 1 SEM.

The TTC estimates of the four large-object scenes that represented different degrees of simulated aliasing were analyzed with a $2 \times 2 \times 3 \times 4$ (gender $\times$ order $\times$ TTC $x$ aliasing) mixed ANOVA. The results indicated that only TTC was significant $\left[F(2,8)=9.51, p<.0325, \omega^{2}=\right.$ $8.22 \%$ ]. The estimated TTC increased as the actual TTC increased; the results of Tukey 's HSD tests indicated that comparisons between $1.1 \mathrm{sec}$ and $4.5 \mathrm{sec}$ or $6.6 \mathrm{sec}$ were significant $(p<.05)$. More important, the absence of an effect of simulated aliasing is consistent with the notion that observers did not extract TTC on a frame-by-frame basis (DeLucia, 1991a).

Further, two-tailed $t$ tests indicated that when TTC was $4.5 \mathrm{sec}$, the mean TTC estimate for the small object, which contained aliasing in $64 \%$ of the frames, was greater than that for the large object that had aliasing in $74 \%$ of the frames $[t(7)=4.887, p<.01]$. When TTC was $1.1 \mathrm{sec}$, this comparison was not significant; however, the mean estimate for the small object, which had $43 \%$ aliasing, was greater than for the large object that had $45 \%$ aliasing $[t(7)=3.103, p<.02]$. The largest value of TTC was not the same for scenes with small and large objects $(6.6 \mathrm{sec}$ vs. $8.5 \mathrm{sec})$; thus, mean TTC estimates were not comparable. These results are consistent with earlier results that indicated that size-arrival effects can occur in absolute TTC judgments (DeLucia, 1991a). A new finding is that mean TTC estimates for small-object scenes can be greater than that for large-object scenes that have aliasing in more frames. This suggests that pictorial size, and not aliasing, underlies size-arrival effects.

In summary, these data suggest that size-arrival effects are not due to aliasing. Further, the degree of irregularity in an object's optical expansion produced by aliasing in computer simulations does not always affect estimates of TTC, probably because observers do not extract TTC on a frame-by-frame basis.

\section{GENERAL DISCUSSION}

The results of Experiment 1 suggest that a conflict between monocular and binocular TTC information does not account for size -arrival effects in active collisionavoidance tasks, at least in the conditions studied here. Although such a result may indicate that passive relative TTC judgments and active collision-avoidance tasks may involve some of the same information or processing strategies that lead to size-arrival effects, future research is needed before such a conclusion can be made.

The results of Experiment 2 suggest that irregularities in optical expansion and TTC information that can occur from computer aliasing do not affect estimates of TTC or account for size-arrival effects. Consistent with the results of a prior study of relative TTC judgments (DeLucia, 1991 a), observers do not seem to extract TTC information on a frame-by-frame basis in a PM task. In models of perceived collision, it is important to determine whether observers use an averaging process to extract TTC and to determine the size of the "averaging window" (see also DeLucia, 1991a; Tresilian, 1993). Further study of the role of aliasing and pictorial size in TTC judgments will provide information about the mechanisms that underlie judgments about collision.

The occurrence of size-arrival effects suggests that optical TTC information (e.g., tau; Lee, 1974) is not sufficient to explain perceived collision and that a more complex model based on multiple sources of information, such as pictorial information, is warranted (DeLucia, 1991a). DeLucia and Warren (1994) considered various explanations of size-arrival effects. One of their conclusions was that neither tau nor pictorial size alone can explain size-arrival effects on collision avoidance, if it is assumed that such information is used continuously over the course of the trial. They proposed that visual information that serves performance varies over time; for example, observers may use pictorial size initially until tau reaches a certain value and contributes more to performance.

It has also been suggested that size-arrival effects may occur because brighter objects can appear closer than dimmer ones, or because larger objects are potentially more dangerous (Hecht, personal communication, March 18, 1998). These ideas deserve further study, although a luminance-based explanation is difficult to apply to black objects (DeLucia \& Warren, 1994).

In prior studies, researchers have also considered whether various tasks that involve TTC judgments are based at least in part on lower order information such as the absolute magnitude of an object's visual angle, the absolute change in visual angle, and the rate of change in visual angle or rate of optical expansion (DeLucia, 199 la; DeLucia \& Novak, 1997; DeLucia \& Warren, 1994; Kebeck \& Landwehr, 1992; Michaels et al., 1997; Regan \& 
Hamstra, 1993; Todd, 1981; Yilmaz \& Warren, 1995). For example, DeLucia and Warren conducted optical analyses that indicated that at the average time of the launch, the values of magnitude of TTC, the square's visual angle, the change in visual angle, and the rate of expansion were not the same for small and for large squares. It seems that participants did not wait for these variables to reach the same value for both squares before jumping. The analyses suggested further that the lower order optical variables (e.g., expansion) may affect the timing of the jump more than TTC information does. They also noted (as did DeLucia, 1991a) that the square's projected size, the change in projected size, and the rate of expansion were relatively less for the smaller objects and that all of these factors could have contributed to performance because their effects were not teased apart. This is an important area for future research.

Finally, it is possible that size-arrival effects occurred with computer simulations because the simulations did not provide some of the most salient information about depth and because the conditions did not represent natural settings (Wann, 1996). To completely address explanations of size-arrival effects based on display artifacts, it is necessary to replicate such effects in the real world (Hecht, personal communication, March 18, 1998), which might pose methodological (and safety) issues, or to determine the minimum features of computer simulations that approximate a natural setting. In any case, on the basis of computer animations of traffic scenes, Caird and Hancock (1994) reported that people typically made greater underestimations of the time at which a large approaching vehicle would reach them as opposed to the times at which smaller vehicles would reach them. This is consistent with a size-arrival effect in what could be considered a more ordinary task.

\section{REFERENCES}

Baker, C. A., \& Steedman, W. C. (1961). Perceived movement in depth as a function of luminance and velocity. Human Factors, 3, 166-173.

CaIRD, J. K., \& HaNCoCK, P. A. (1994). The perception of arrival time for different oncoming vehicles at an intersection. Ecological Psychology, 6, 83-109.

Cavallo, V., \& Laurent, M. (1988). Visual information and skill level in time-to-collision estimation. Perception, 17, 623-632.

Delucia, P. R. (1989). Pictorial depth cues and motion-produced information for depth perception. Dissertation Abstracts International, 1526, 51-03B. (University Microfilms No. 90-20517)

DeLuCia, P. R. (1991a). Pictorial and motion-based information for depth perception. Journal of Experimental Psychology: Human Perception \& Performance, 17, 738-748.

DELUCIA, P. R. (1991b). Small near objects can appear farther than large far objects during object motion and self motion: Judgments of object-self and object-object collisions. In P. J. Beek, R. J. Bootsma, \& P. C. W. van Wieringen (Eds.), Studies in perception and action: Posters presented at the VIth International Conference on Event Perception and Action (pp. 94-100). Amsterdam: Rodopi.

DELuCIA, P. R. (1995). Effects of pictorial relative size and groundintercept information on judgments about potential collision in perspective displays. Human Factors, 37, 528-538.

DeLuCIA, P. R., \& LidDelL, G. W. (1998). Cognitive motion extrapolation and cognitive clocking in prediction motion tasks. Journal of
Experimental Psychology: Human Perception \& Performance, 24, 901-914.

DeLuCiA, P. R., \& Novak, J. B. (1997). Judgments of relative time-tocontact of more than two approaching objects: Toward a method. Perception \& Psychophysics, 59, 913-928.

Delucia, P. R., \& Warren, R. (1994). Pictorial and motion-based depth information during active control of self-motion: Size-arrival effects on collision avoidance. Journal of Experimental Psychology: Human Perception \& Performance, 20, 783-798.

GraY, R., \& REGAN, D. (1996). Estimates of time to collision based on binocular and monocular visual information [Abstract]. Investigative Ophthalmology \& Visual Science, 37, S652.

Gray, R., \& REGAN, D. (1998). Accuracy of estimating time to collision using binocular and monocular information. Vision Research, 38, 499-512.

Gray, R., \& ReGan, D. (1999). Motion in depth: Adequate and inadequate simulation. Perception \& Psychophysics, 61, 236-245.

Hecht, H., Kaiser, M. K., Savelsbergh, G. J. P., \& van der Kamp, J. (1996, November). The effect of time-sampling on temporal range judgments in real and simulated environments. Paper presented at the 37th Annual Meeting of the Psychonomic Society, Chicago.

Heuer, H. (1993). Estimates of time to contact based on changing size and changing target vergence. Perception, 22, 549-663.

HiLLs, B. L. (1975). Some studies of movement perception, age and accidents (Rep. No. SR 137). Crowthorne, England: Department of the Environment, Transport and Road Research Laboratory.

HOCHBERG, J. (1986). Representation of motion and space in video and cinematic displays. In K. R. Boff, L. Kaufman, J. P. Thomas (Eds.), Handbook of perception and human performance (pp. 22-1 to 22-64). Toronto: Wiley.

KatSER, M. K., \& MowafY, L. (1993). Optical specification of time-topassage: Observers' sensitivity to global tau. Journal of Experimental Psychology: Human Perception \& Performance, 19, $1028-1040$.

KEBECK, G., \& LANDWEHR, K. (1992). Optical magnification as event information. Psychological Research, 54, 146-159.

LEE, D. N. (1974). Visual information during locomotion. In R. B. MacLeod \& H. L. Pick, Jr. (Eds.), Perception: Essays in honor of James J. Gibson (pp. 250-267). Ithaca, NY: Cornell University Press.

LEE, D. N. (1980). The optic flow field: The foundation of vision. Philosophical Transactions of the Royal Society of London: Series B, 290. 169-179.

Michaels, C. F., Zeinstra, E., \& Oudeians, R. R. D. (1997). Information and action in timing the punching of a falling ball [Abstract]. In G. L. Torenvliet \& K. J. Vicente (Eds.), Ninth International Conference on Perception and Action: Proceedings (p. 16). Scarborough, ON: University of Toronto, International Society for Ecological Psychology.

Morgan, M. J. (1980). Analogue models of motion perception. Philosophical Transactions of the Roval Societv of London: Series B, 290, $117-135$

REGAN, D. (1997). Visual factors in hitting and catching. Journal of Sports Sciences, 15, 533-558.

Regan, D., \& Hamstra, S. J. (1993). Dissociation of discrimination thresholds for time to contact and for rate of angular expansion. $\mathrm{Vi}$ sion Research, 33, 447-462.

Savelsbergh, G. J. P., Whiting, H. T. A., \& Bootsma, R. J. (1991). Grasping tau. Journal of Experimental Psychology: Human Perception \& Performance, 17, 315-322.

SCHIFF, W., \& DeTWILER, M. L. (1979). Information used in judging impending collision. Perception, 8, 647-658.

SchIFF, W., \& OLDAK, R. (1990). Accuracy of judging time to arrival: Effects of modality, trajectory, and gender. Journal of Experimental Psychology: Human Perception \& Performance, 16, 303-316.

SmITH, W. M., \& Gulick, W. L. (1957). Dynamic contour perception. Journal of Experimental Psychology, 33, 145-151.

ToDD, J. T. (1981). Visual information about moving objects. Journal of Experimental Psychology: Human Perception \& Performance, 7. 795-810.

Tresilian, J. R. (1993). Four questions of time-to-contact: A critical analysis of research into interceptive timing. Perception, 22, 653-680.

Tresilian, J. R. ( 1994). Perceptual and motor processes in interceptive timing. Human Movement Science, 13, 335-373. 
Tresilian, J. R. (1995). Perceptual and cognitive processes in time-tocontact estimation: Analysis of prediction-motion and relative judgment tasks. Perception \& Psychophysics, 57, 231-245.

VinCENT, A., \& REgAN, D. (1997). Judging the time to collision with a simulated textured object: Effect of mismatching rate of expansion of object size and of texture element size. Perception \& Psychophysics, 59, 32-36.

WANN, J. P. (1996). Anticipating arrival: Is the tau margin a specious theory? Journal of Experimental Psychology: Human Perception \& Performance, 22, 1031-1048.

WATT, A. (1989). Fundamentals of three-dimensional computer graphics. Wokingham, England: Addison-Wesley.

YIL.MAZ, E. H., \& WARREN, W. H. (1995). Visual control of braking: A test of the tau-dot hypothesis. Journal of Experimental Psychology: Human Perception \& Performance, 21, 996-1014.

\section{NOTES}

1. Here, aliasing refers to effects of the computer's digitization process rather than to technical meanings assigned by signal-analysis theory (see Watt, 1989).

2. Informal observations indicated that the simulation of self-motion was not compelling and that the displays appeared more as object motion. (Indeed, only 4 of the 16 observers in Experiment I reported that the display appeared as self-motion rather than as object motion.) Thus, observers were told that the displays simulated an environment in which they were either moving toward a stationary square at a constant speed or stationary and the square moved toward them. The occurrence of size-arrival effects suggests that this factor is not critical.

3. The present study does not rule out the possibility that lines of constant thickness indicated to observers that the square (self) was stationary (i.e., had an infinite TTC value, see Gray \& Regan, 1999) and, as suggested by an anonymous reviewer, presented a conflict between the TTC specified by the square"s "texture" and the TTC specified by the overall expansion (area) of the square. Owing to technical limitations, it was not possible to use filled-in squares. Gray and Regan (1999; see also Vincent \& Regan, 1997) reported that errors in TTC estimates can increase (in the direction of overestimation) when a simulated approaching object contains texture elements (dots) that remain constant in optical size, compared with errors made when elements expand in accordance with the object 's optical expansion. However, this effect decreased as dot size decreased. When the optical size of the dots was 2.2-4.4 min of arc, errors were comparable, whether dot size expanded or remained constant (other dot sizes were $6.0,7.6$, and $10.5 \mathrm{~min}$ of arc). In the present study, the thickness (width) of the vertical lines subtended about $3.58 \mathrm{~min}$ of arc; the thickness (height) of the horizontal lines was about
$4.91 \mathrm{~min}$ of arc. Such angular extents may be sufficiently small to minimize the potential effects of constant line thickness on TTC estimation. Further, unlike Gray and Regan's (1999) dots, the lines in this study expanded along one dimension during approach. Moreover, comparisons of TTC estimates to specific psychophysical models of visual processing of image expansion and perception of motion in depth suggest that greater weight is given to object expansion than to expansion of texture element size (Gray \& Regan, 1999). If the reverse were true in the present study, we would expect observers to overestimate TTC and to jump later than the means indicate. Nevertheless, further investigation is needed to determine whether the present results are limited to displays with lines of constant thickness.

4. The time histories appeared noisier than in DeLucia and Warren (1994); however, they used a sampling rate of only $10 \mathrm{~Hz}$.

5. In DeLucia and Warren's (1994) study, altitude was sampled at $10 \mathrm{~Hz}$ and launch time was defined as the time at which the slope of the altitude exceeded $1 \%$. In the present study, altitude was sampled at $35 \mathrm{~Hz}$; thus, launch time was defined as the time at which the slope of the altitude exceeded $29 \%$.

6. If a maximum altitude was reached and then remained the same in successive frames, the time and altitude on the last frame (before descent) was used.

7. Probability values from the results of the ANOVAs reflect Greenhouse-Geisser corrections.

8. The responses of five observers seemed unusual on many trials and/or suggested that they did not follow the instructions (e.g., did not always descend after the jump or reached peak altitude and stopped before reaching the square). It was difficult to derive objective criteria to exclude such observers, because many observers had at least one trial that seemed unusual. Therefore, additional analyses were conducted in which the five observers were replaced with results from new observers. The analyses of monocular conditions were similar to those in Experiment 1 , except that there was an interaction between square top and square size on launch altitude $\left(\rho<.0154, \omega^{2}=2.40 \%\right)$ and the percentage of crashes was greater with the 128 - $\mathrm{ft}$ square than with the 16 ft square $(p<.05)$. The results of binocular conditions were similar to those in Experiment 1, except that there was an interaction between square top and square size on time at peak altitude $\left(p<.0317, \omega^{2}=\right.$ $2.77 \%$ ). The results of combining monocular and binocular conditions again indicated no effects involving ocularity.

9. I determined only whether there was any change in the object's optical size (either width or height), but not whether the amount of change was correct.

(Manuscript received December 18, 1997; revision accepted for publication June 26,1998 .) 